\title{
Coronavirus Disease 2019 Coagulopathy: Disseminated Intravascular Coagulation and Thrombotic Microangiopathy-Either, Neither, or Both
}

\author{
Marcel Levi, MD, PhD, FRCP ${ }^{1,2}$ Jecko Thachil, MD, FRCP 3 \\ ${ }^{1}$ Department of Medicine, University College London Hospitals NHS \\ Foundation Trust, London, United Kingdom \\ 2 NIHR UCLH/UCL Biomedical Research Centre, London, United Kingdom \\ ${ }^{3}$ Department of Haematology, Manchester Royal Infirmary, \\ Manchester, United Kingdom
}

Semin Thromb Hemost 2020;46:781-784.

Patients with severe coronavirus disease 2019 (COVID-19) infections manifest coagulation abnormalities that have been associated with respiratory deterioration and death. ${ }^{1,2}$ In addition, many patients with severe COVID-19 infections develop venous thromboembolism, which seems to be related to the coagulopathy. ${ }^{3}$ It has also been suggested that undiagnosed pulmonary embolism contributes to a sudden deterioration of pulmonary oxygen exchange that is sometimes seen in patients with COVID-19 infections. ${ }^{4,5}$

The coagulopathy associated with COVID-19 mimics other systemic coagulopathies that are regularly seen in severe infections, such as disseminated intravascular coagulation (DIC) or thrombotic microangiopathy (TMA) ${ }^{6-8}$ However, at the same time, the clinical and laboratory characteristics of the coagulation changes in COVID-19 are distinctly different from the common presentation of these conditions.

\section{Coagulation Tests in COVID-19}

The most striking coagulation test abnormality in severe COVID-19 patients is an abnormally high D-dimer level. ${ }^{1,2,9,10}$ In several series of patients with COVID-19, markedly increased D-dimer levels were observed in almost $50 \%$ of patients. Also, patients with D-dimer levels more than sixfold the upper limit of normal were found to have an increased need for mechanical ventilation and a significantly higher risk of death.

Another coagulation abnormality that is seen in the most severe patients is thrombocytopenia. ${ }^{11,12}$ Most of these patients have a platelet count between 100 and $150 \times 10^{9} / \mathrm{L}$, and lower platelet counts are rarely $(<5 \%)$ seen. ${ }^{9,13}$ In contrast to thrombocytopenia associated with other infections including viral disease and bacterial sepsis, ${ }^{14}$ a low platelet count in COVID-19 has not been significantly associated with an ad-

published online June 8,2020
Issue Theme Maintaining Hemostasis and Preventing Thrombosis in COVID-19 -Part I; Guest Editors: Emmanuel J. Favaloro, PhD, FFSc (RCPA), and Giuseppi Lippi, MD.
Address for correspondence Marcel Levi, MD, Department of Medicine, University College London Hospitals NHS Foundation Trust, 250 Euston Road, London NW1 2PG, United Kingdom (e-mail: marcel.levi@nhs.net).

verse outcome, although very low platelet counts can be an exception. ${ }^{11}$

The prothrombin time in patients with more severe COVID19 infection is only mildly prolonged ( 3 seconds). ${ }^{2,15}$ Prolongation of the activated partial thromboplastin time (aPTT) is less clear and might be obscured by (very) high levels of factor VIII and fibrinogen that instead sometimes leads to shortening of the aPTT.

Mean fibrinogen plasma concentration in COVID-19 patients are strikingly high, which is likely due to an acute phase response. ${ }^{2}$ However, a swift drop in plasma fibrinogen to concentrations below $<1.0 \mathrm{~g} / \mathrm{L}$ was demonstrated in a small number of the most severe COVID-19 patients in China, shortly before they died.

Plasma levels of protease inhibitor such as protein C and antithrombin are slightly decreased, in particular in the nonsurviving patients, but plasma concentrations seldom fall below $80 \%$ of normal values. ${ }^{2}$

\section{Is this Disseminated Intravascular Coagulation?}

The combination of increased D-dimer, thrombocytopenia, and prolonged global coagulation tests mimics the pattern seen in DIC. ${ }^{6}$ However, there seem to be distinct differences from DIC commonly seen in patients with sepsis, cancer, or other underlying conditions known to be associated with DIC. First, in most cases with DIC complicating a serious systemic condition, a more profound thrombocytopenia is observed. ${ }^{16}$ Also, these patients usually have much lower levels of coagulation factors (in particular factors II, V, VII, and $\mathrm{X}$ ) and severely decreased plasma concentrations of physiological anticoagulants such as antithrombin and protein C. ${ }^{17}$
Copyright $\odot 2020$ by Thieme Medical Publishers, Inc., 333 Seventh Avenue, New York, NY 10001, USA. Tel: +1(212) 760-0888.
DOI https://doi.org/ 10.1055/s-0040-1712156. ISSN 0094-6176. 
One of the pathogenetic hallmarks of DIC is dysregulated thrombin generation, usually as a result of tissue factor expression on mononuclear and possibly endothelial cells induced by proinflammatory cytokines, most prominently interleukin (IL)-6. ${ }^{18}$ While IL-6 and other pro-inflammatory cytokines were shown to be extremely elevated in severe COVID-19 infection, there is not yet definitive proof of excessive thrombin generation in these patients. Observations with a thrombin generation test and assessment of markers for prothrombin to thrombin activation $(\mathrm{F} 1+2)$ and thrombin-antithrombin complexes would be helpful in obtaining further insight into this issue.

In view of the coagulation test results, most patients with COVID-19 would not reach a score sufficiently high to be diagnosed with overt DIC according to the DIC score of the International Society on Thrombosis and Haemostasis (ISTH). ${ }^{19}$ The clinical presentation of the COVID-19 coagulopathy is mostly prothrombotic, with a high incidence of overt venous (and possibly arterial) thromboembolism, and not many hemorrhagic complications, which is no surprise in the absence of a real consumption coagulopathy.,20

Taken together, it might be fair to conclude that the coagulopathy of COVID-19 should be classified as a specific form of intravascular coagulation, which is distinctly different from DIC commonly seen in other conditions and may need new diagnostic criteria.

\section{Is this Thrombotic Microangiopathy?}

TMA is a result of increased platelet adhesion to the vascular endothelium in association with platelet aggregation and activation, causing consumptive thrombocytopenia. ${ }^{8}$ The resultant platelet thrombi in the microvasculature cause impaired organ function and classically contribute to complications such as renal insufficiency or neurological disease as well as microangiopathic hemolysis. ${ }^{21}$

Histopathology from postmortem examinations in COVID-19 patients has shown typical microvascular plateletrich thrombotic depositions in small vessels of the lungs along the foci of local hemorrhage and accumulation and entrapment of inflammatory cells, such as neutrophils, in alveolar capillaries. This picture is compatible with pulmonary TMA. There was not a lot of evidence for microvascular platelet-rich thrombi in other organs. ${ }^{22}$

Overt intravascular hemolysis and severe thrombocytopenia, as seen in typical cases of thrombocytopenic thrombotic purpura (TTP), is not a clinical feature of COVID-19 infection. However, some other laboratory abnormalities in severe COVID-19 that may point to a form of TMA are an increased LDH and, in some patients, strikingly high ferritin levels, in particular in patients with the most severe organ dysfunction. ${ }^{1}$

Pathologically increased platelet-vessel wall interaction as seen in TMA is caused by the presence of ultra-large von Willebrand factor multimers released from injured endothelial cells. ${ }^{8}$ Under physiological circumstances, these multimers would be cleaved by ADAMTS-13 (a disintegrin and metalloprotease with a thrombospondin type I motif-13).
However, COVID-19 infection with associated endothelial cell perturbation may lead to decreased ADAMTS-13 levels due to consumption or proteolytic degradation, resulting in high levels of uncleaved von Willebrand factor multimers and ensuing deposit of microvascular platelet thrombi. This mechanism has been shown to occur in other severe infectious conditions. ${ }^{23,24}$ Currently, there are no data on ADAMTS-13 levels in severe COVID-19 infection.

Taken together, there is evidence of a localized TMA as evidenced by microvascular thrombosis in COVID-19-affected lungs, but there is no strong evidence for a systemic TMA.

\section{Pathogenetic Pathways Specific for COVID- 19 Coagulopathy}

Coronavirus infections seem to be associated with a typical activation of the fibrinolytic system. Experiments in mice with a targeted deletion of urokinase-type plasminogen activator indicated that the urokinase-driven pathway was an important mediator of lethality. ${ }^{25}$ Moreover, patients with human SARS-CoV-1 infection had sixfold increased plasma levels of t-PA (tissue-type plasminogen activator). ${ }^{26}$ Presumably, the COVID-19 inflammation-induced endothelial cell injury may cause a massive release of plasminogen activators. The resulting plasmin generation may be an important factor explaining the high levels of D-dimer seen in patients with severe COVID-19 infection. In addition, plasmin-mediated effects on the metalloproteinase system may result in changes in the extracellular matrix, facilitating capillary leakage and pulmonary edema. ${ }^{27}$

There is a strong connection between bronchoalveolar coagulation and fibrinolysis and the pathogenesis of acute respiratory distress syndrome (ARDS), in which enhanced intrapulmonary fibrin deposition due to abnormal bronchoalveolar fibrin turnover is a crucial factor. ${ }^{28}$ Measurement of coagulation and fibrinolysis factors in the bronchoalveolar fluid have demonstrated that enhanced intrapulmonary thrombin generation, insufficiently balanced by physiological anticoagulant factors and endogenous fibrinolysis, is mediating this pathogenetic pathway. ${ }^{29}$ The clinical and laboratory picture of serious ARDS in ventilated COVID-19 patients and important coagulopathic changes point to an important role of bronchoalveolar and pulmonary vascular fibrin turnover in the most severe patients.

\section{Conclusion}

Severe COVID-19 infection is associated with coagulation abnormalities that encompass elements reminiscent of both DIC and TMA (-Fig. 1). However, this coronavirus infection seems to cause a specific coagulopathy that is more localized and distinctly different from "classical" DIC or TMA syndromes. The specific coagulopathic features of this disease are likely relevant in view of the high incidence of thromboembolic complications in severely affected patients and may present an important point of impact for (supportive) preventive or therapeutic management strategies to improve the clinical outcome. 


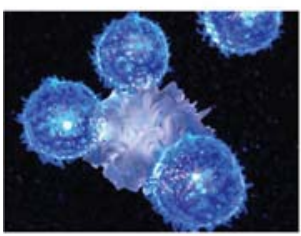

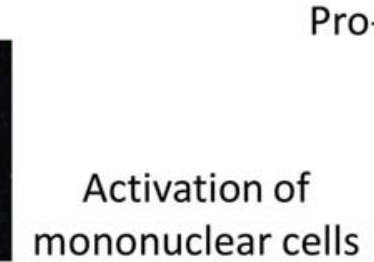<smiles>c1ccccc1</smiles>

Tissue factor-driven activation of intravascular coagulation

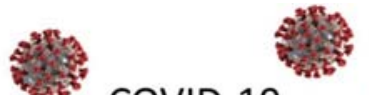

के

COVID-19

I

Pro-inflammatory

cytokines

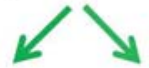

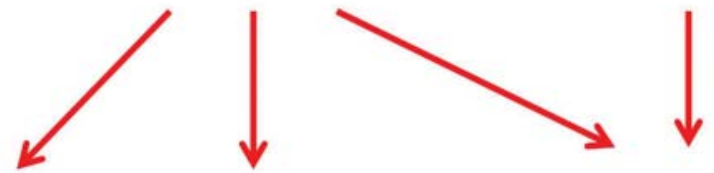

Thrombo-embolic events

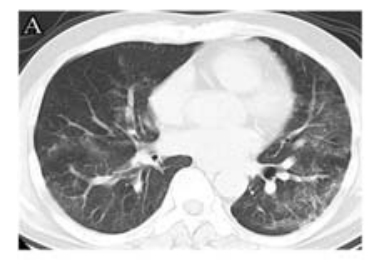

Broncho-alveolar fibrin deposition and acute lung injury

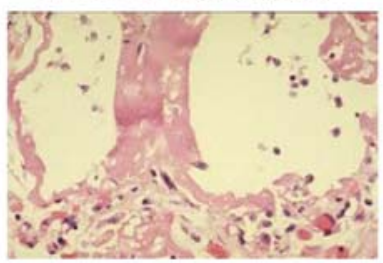

High D-dimer levels

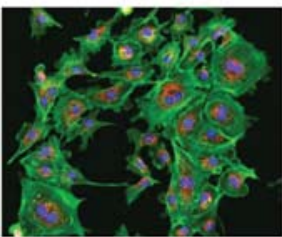
injury

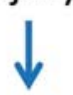

Plasminogen activator release and plasmin generation

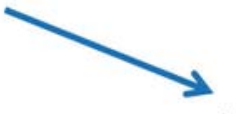

Fig. 1 Schematic representation of pathogenetic pathways in COVID-19 coagulopathy. A COVID-19 infection causes upregulation of proinflammatory cytokines, resulting in mononuclear cell activation and injury to the endothelium. This may lead to a systemic procoagulant response, hypothetically contributing to an increased incidence of venous thromboembolism and abnormal fibrin turnover and deposition, which is a hallmark of acute lung injury. Endothelial cell perturbation in coronavirus infection also leads to release of plasminogen activators that in combination with the procoagulant response may be responsible for the (very) high D-dimer levels. Release of ultralarge von Willebrand factor multimers, insufficiently matched by the cleaving capacity of ADAMTS-13 (a disintegrin and metalloprotease with a thrombospondin type I motif-13), may lead to local pulmonary microvascular thrombosis. COVID-19, coronavirus disease 2019.

Conflicts in Interest

None.

\section{References}

1 Zhou F, Yu T, Du R, et al. Clinical course and risk factors for mortality of adult inpatients with COVID-19 in Wuhan, China: a retrospective cohort study. Lancet 2020;395(10229):1054-1062

2 Tang N, Li D, Wang X, Sun Z. Abnormal coagulation parameters are associated with poor prognosis in patients with novel coronavirus pneumonia. J Thromb Haemost 2020;18(04):844-847

3 Klok FA, Kruip MJHA, van der Meer NJM, et al. Incidence of thrombotic complications in critically ill ICU patients with COVID-19. Thromb Res 2020 (e-pub ahead of print). Doi: 10.1016/j.thromres.2020.04.013

4 Yuanliang X, Wang X, Yang P, Zhang S. COVID-19 complicated by acute pulmonary embolism. Radiology: Cardiothoracic Imaging 2020; 2(02):e200067 (e-pub ahead of print). Doi: 10.1148/ryct.2020200067

5 Danzi GB, Loffi M, Galeazzi G, Gherbesi E. Acute pulmonary embolism and COVID-19 pneumonia: a random association?
Eur Heart J 2020 (e-pub ahead of print) . Doi: 10.1093/eurheartj/ehaa254

6 Gando S, Levi M, Toh CH. Disseminated intravascular coagulation. Nat Rev Dis Primers 2016;2:16037

7 Levi M, Ten Cate H. Disseminated intravascular coagulation. N Engl J Med 1999;341(08):586-592

8 George JN, Nester CM. Syndromes of thrombotic microangiopathy. N Engl J Med 2014;371(07):654-666

9 Huang C, Wang Y, Li X, et al. Clinical features of patients infected with 2019 novel coronavirus in Wuhan, China. Lancet 2020;395 (10223):497-506

10 Lippi G, Favaloro EJ. D-dimer is associated with severity of coronavirus disease 2019: a pooled analysis. Thromb Haemost 2020;120(05):876-878

11 Lippi G, Plebani M, Henry BM. Thrombocytopenia is associated with severe coronavirus disease 2019 (COVID-19) infections: a meta-analysis. Clin Chim Acta 2020;506: 145-148

12 Brogaard Larsen J, Pasalic L, Hvas AM. Platelets in coronavirus disease. Semin Thromb Hemost 2020;46(07):823-825 
13 Guan WJ, Ni ZY, Hu Y, et al; China Medical Treatment Expert Group for Covid-19. Clinical characteristics of coronavirus disease 2019 in China. N Engl J Med 2020;46:823-825

14 Akca S, Haji-Michael P, de Mendonça A, Suter P, Levi M, Vincent JL. Time course of platelet counts in critically ill patients. Crit Care Med 2002;30(04):753-756

15 Henry BM, Lippi G, Plebani M. Laboratory abnormalities in children with novel coronavirus disease 2019. Clin Chem Lab Med 2020 (e-pub ahead of print) . Doi: 10.1515/cclm-20200272

16 Levi M. Platelets in critical illness. Semin Thromb Hemost 2016;42 (03):252-257

17 Levi M, Sivapalaratnam S. Disseminated intravascular coagulation: an update on pathogenesis and diagnosis. Expert Rev Hematol 2018;11(08):663-672

18 Levi M, van der Poll T, Büller HR. Bidirectional relation between inflammation and coagulation. Circulation 2004;109(22): 2698-2704

19 Taylor FB Jr, Toh CH, Hoots WK, Wada H, Levi M; Scientific Subcommittee on Disseminated Intravascular Coagulation (DIC) of the International Society on Thrombosis and Haemostasis (ISTH). Towards definition, clinical and laboratory criteria, and a scoring system for disseminated intravascular coagulation. Thromb Haemost 2001;86(05):1327-1330

20 Tang N, Bai H, Chen X, Gong J, Li D, Sun Z. Anticoagulant treatment is associated with decreased mortality in severe coronavirus disease 2019 patients with coagulopathy. J Thromb Haemost 2020 (e-pub ahead of print) . Doi: 10.1111/jth.14817
21 Scully M, Goodship T. How I treat thrombotic thrombocytopenic purpura and atypical haemolytic uraemic syndrome. Br J Haematol 2014;164(06):759-766

22 Fox SE, Atmakbekov A, Harbert JL, Li G, Brown JQ, Vander Heide RS. Pulmonary and cardiac pathologyin Covid-19: the first autopsy series from New Orleans. MedRxiv 2020 (e-pub ahead of print) Doi: $10.1101 / 2020.04 .06 .20050575$

23 Levi M, Scully M, Singer M. The role of ADAMTS-13 in the coagulopathy of sepsis. J Thromb Haemost 2018;16(04):646-651

24 Schwameis M, Schörgenhofer C, Assinger A, Steiner MM, Jilma B. VWF excess and ADAMTS13 deficiency: a unifying pathomechanism linking inflammation to thrombosis in DIC, malaria, and TTP. Thromb Haemost 2015;113(04):708-718

25 Gralinski LE, Bankhead A III, Jeng S, et al. Mechanisms of severe acute respiratory syndrome coronavirus-induced acute lung injury. MBio 2013;4(04):e00271-e13

26 Liu ZH, Wei R, Wu YP, et al. Elevated plasma tissue-type plasminogen activator (t-PA) and soluble thrombomodulin in patients suffering from severe acute respiratory syndrome (SARS) as a possible index for prognosis and treatment strategy. Biomed Environ Sci 2005;18(04):260-264

27 Lijnen HR. Matrix metalloproteinases and cellular fibrinolytic activity. Biochemistry (Mosc) 2002;67(01):92-98

28 Bellingan GJ. The pulmonary physician in critical care * 6 : the pathogenesis of ALI/ARDS. Thorax 2002;57(06):540-546

29 Glas GJ, Van Der Sluijs KF, Schultz MJ, Hofstra JJ, Van Der Poll T, Levi M. Bronchoalveolar hemostasis in lung injury and acute respiratory distress syndrome. J Thromb Haemost 2013;11(01):17-25 